\title{
MicroRNA-129-2-3p directly targets Wipl to suppress the proliferation and invasion of intrahepatic cholangiocarcinoma
}

\author{
Chen Chen ${ }^{1 \#, ~ J i n q i o n g ~ J i a n g 2 \#, ~ M e n g ~ F a n g ~}{ }^{1}$, Lei Zhou ${ }^{1}$, Yongzhi Chen ${ }^{1}$, Jia Zhou ${ }^{1}$, Yinghui Song ${ }^{1}$, Gaoying

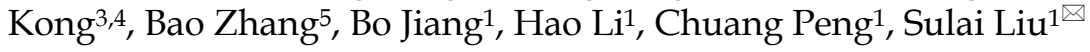 \\ 1. Department of Hepatobiliary Surgery, Hunan Provincial People's Hospital/The First Affiliated Hospital of Hunan Normal University, Changsha, Hunan \\ Province, People's Republic of China. \\ 2. Department of Oncology, Hunan Provincial People's Hospital/The First Affiliated Hospital of Hunan Normal University, Changsha, Hunan Province, \\ People's Republic of China. \\ 3. Department of Anesthesiology, Hunan Provincial People's Hospital/The First Affiliated Hospital of Hunan Normal University, Changsha, Hunan Province, \\ People's Republic of China. \\ 4. Clinical Research Center for Anesthesiology of ERAS in Hunan Province, Changsha 410005, China. \\ 5. Department of Minimally Invasive Surgery, The Second People's Hospital of Hunan Province, Changsha 410017, China. \\ \#This author contributes equally to this work.
}

$\triangle$ Corresponding author: Sulai Liu, PhD, M.D., Department of Hepatobiliary Surgery, Hunan Provincial People's Hospital/The First Affiliated Hospital of Hunan Normal University, Changsha, Hunan Province, People's Republic of China. Tel/fax: 08673183929520. E-mail: liusulai@hunnu.edu.cn.

() The author(s). This is an open access article distributed under the terms of the Creative Commons Attribution License (https://creativecommons.org/licenses/by/4.0/). See http://ivyspring.com/terms for full terms and conditions.

Received: 2019.10.25; Accepted: 2020.02.09; Published: 2020.03.05

\begin{abstract}
Accumulated studies showed that numerous microRNAs (miRNAs) were aberrantly expressed in human intrahepatic cholangiocarcinoma (ICC) and contributed to the tumorigenic processes. However, whether miR-129-2-3p is implicated in the ICC initiation and progression is still limited. Here, the results revealed that miR-129-2-3p expression was notably decreased in ICC tissues and cell lines, and that a low miR-129-2-3p expression was obviously associated with distant metastasis and clinical stage. Exogenous miR-129-2-3p expression evidently repressed the proliferative and invasive abilities of ICC cells. Mechanistic studies indicated that Wild-type p53-induced phosphatase 1 (Wipl) was a direct target gene for miR-129-2-3p in ICC cells. Furthermore, silencing Wipl expression mimicked the suppressive effects of miR-129-2-3p upregulation on ICC cells. Interestingly, reintroduction of Wipl expression partially abolished the miR-129-2-3p -reduced cell proliferation and invasion in ICC. Moreover, ectopic miR-129-2-3p expression hindered the ICC tumor growth in vivo. To the best of our knowledge, it is the first time to reveal that miR-129-2-3p plays a crucial role in tumor suppression in ICC pathogenesis through directly targeting Wipl. These results will aid in elucidating the roles of miR-129-2-3p in ICC, and suggest that this miRNA may provide a potential target for the treatment of ICC
\end{abstract}

Key words: miR-129-2-3p involved ICC via Wip1

\section{Introduction}

Intrahepatic cholangiocarcinoma (ICC) is an aggressive and poorly understood biliary malignancy that is frequently diagnosed at advanced stages, which limits its treatment options [1]. In recent years, the incidence and mortality of ICC has drastically increased with geographic variation [2,3]. Furthermore, the long-term survival of patients with unresectable ICC is dismal, with less than $5 \%$ to $10 \%$ of patients surviving at 5 years after the diagnosis $[2,4]$. Surgical resection remains the only approach with curative intent, achieving a 5-year survival rate of approximately $20 \%$ at early $\mathrm{T} 1-\mathrm{T} 2$ stages; Still worse, in sharp contrast to the use of standard therapies for patients with advanced lung/breast/ 
colorectal cancers and hepatocellular carcinoma, systemic chemotherapy and molecular-targeted therapies have had limited success in treating ICC [5]. Therefore, it is imperative to fully elucidate the mechanisms responsible for ICC carcinogenesis and progression, as they may provide novel and effective strategic approaches to improve the prognosis of ICC patients.

Recently, microRNAs was shown to implicated in controlling multiple steps of ICC onset and progression [6]. Aberrant expression of the miR-129$2-3 p$ has been detected in multiple types of carcinoma, yet its expression and potential biologic role in ICC has not been investigated [7-9].

Wip1 (also known as PPM1D) is a monomeric serine/threonine phosphatase of the PP2C family, and its expression is increased after DNA damage, which inactivates p53 and promotes termination of the DDR pathway [10,11]. Wip1 dephosphorylates many proteins, including ataxia-telangiectasia mutated (ATM), Chk1, Chk2, p53, p38 and Mdm2 [10]. These proteins that belong to DNA damage response markers are often decreased in DNA damage response pathways, which contribute to tumor progression. Others and our previous studies showed that Wip1 were over expression in a wide range of tumor tissues including kidney [12], breast [13], lung [14], ovarian [15], neuroblastoma [16]. Our previous research showed that Wip1 was highly expressed in ICC tissues and cell lines and may be a key regulator in the tumorigenicity and invasion of human ICC $[17,18]$. However, the potential mechanism is unknown. In current study, we decided to investigate the effects of miR-129-2-3p in ICC and have demonstrated that miR-129-2-3p could inhibit cell invasion and migration via Wip1 in ICC.

\section{Materials and methods}

\section{Clinical tissue specimens}

In total, 90 patients with ICC who received surgical excision in Hunan Provincial People's Hospital/The First Affiliated Hospital of Hunan Normal University were recruited between Autumn 2016 and May 2017. None of the patients received any anticancer therapies before surgery, including chemotherapy, radiotherapy or immunotherapy. Following surgical resection, tumor specimens were quickly frozen in liquid nitrogen and then stored at $-80^{\circ} \mathrm{C}$. Prior written informed consent was provided from all participants and this study was approved by the Ethics Committee of Hunan Provincial People's Hospital/The First Affiliated Hospital of Hunan Normal University.

\section{Cell culture and transient transfection}

A panel of human ICC cell lines, including QBC-939, RBE, as well as a normal human biliary epithelial cell (BEC) were purchased from the Type Culture Collection of the Chinese Academy of Sciences (Shanghai, China). Cells were incubated under the conditions of $5 \% \quad \mathrm{CO}_{2}$ and $37^{\circ} \mathrm{C}$ in Dulbecco's modified Eagle's medium (DMEM) containing 10\% fetal bovine serum (FBS; both from Gibco, Invitrogen, Carlsbad, CA, USA) and $1 \%$ penicillin/ streptomycin mixture (Sigma-Aldrich, St. Louis, MO).

The synthetic miR-129-2-3p mimics and miRNA mimics negative control (miR-NC) were obtained from Guangzhou RiboBio Co, Ltd (Guangzhou, Guangdong, China). The Wip1-overexpression plasmid was generated by inserting Wip1 cDNA lacking its 3'-UTR into a pCMV vector. This plasmid was chemically produced by Shanghai GenePharma Co., Ltd (Shanghai, China). The siRNA-Wip1 (si-Wip1) was obtained from Qiagen $\mathrm{GmbH}$ (Hilden, Germany) and used to knock down the endogenous expression of Wip1. A control siRNA (si-NC) served as a negative control for si-Wip1. RNA oligonucleotides and plasmid were transfected into cells using Lipofectamine 2000 (Invitrogen; Thermo Fisher Scientific, Inc, Waltham, MA, USA) following the manufacturer's protocol.

\section{RNA extraction and reverse transcription- quantitative polymerase chain reaction (RT-qPCR)}

TRIzol reagent (Invitrogen; Thermo Fisher Scientific, Inc.) was utilized for the isolation of total RNA from tissue specimens and cells. The concentration of total RNA was determined with a Nanodrop 2000 (Thermo Fisher Scientific, Waltham, MA). Total RNA was reversely transcribed into cDNA using the miScript Reverse Transcription kit (Qiagen $\mathrm{GmbH}$, Hilden, Germany). After that, quantitative PCR (qPCR) was carried out to detect miR-129-2-3p expression with the miScript SYBR Green PCR kit (Qiagen $\mathrm{GmbH}$ ). For the determination of Wip1 mRNA, reverse transcription was carried out using the PrimeScript RT reagent kit (Takara Bio, Dalian, China). Next, qPCR was conducted through a SYBR Premix Ex Taq ${ }^{\mathrm{TM}}$ Kit (Takara Bio) and an Applied Biosystems 7500 Real-time PCR System (Thermo Fisher Scientific, Waltham, MA). U6 served as the internal reference for miR-129-2-3p, and GAPDH was the internal control for Wip1. Relative gene expression was analyzed using the $2^{-\Delta \Delta \mathrm{Cq}}$ method [19]. 


\section{Cell Counting Kit-8 (CCK-8) assay}

Transfected cells were plated into 96-well plates at a density of $3 \times 10^{3}$ cells per well. Five replicate wells were used for every group. After culture for 0, 24, 48, and $72 \mathrm{~h}, \mathrm{CCK}-8$ assay was carried out by inoculating $10 \mu \mathrm{L}$ of CCK-8 reagent (Dojindo Molecular Technologies, Inc., Kumamoto, Japan) into each well. Cells were incubated at $37{ }^{\circ} \mathrm{C}$ with $5 \% \mathrm{CO}_{2}$ for additional $2 \mathrm{~h}$, and the absorbance in each well was measured using a spectrophotometric plate reader (Infinite ${ }^{\circledR} 200$ PRO; Tecan Group, Ltd., Mannedorf, Switzerland).

\section{Transwell cell invasion assay}

Transwell chamber (Costar ${ }^{\mathrm{TM}}$; Corning, Inc., Corning, NY, USA) precoated with Matrigel (BD Biosciences, San Jose, CA) was applied to assess the cellular invasive capacity. In detail, the transfected cells were harvested after $48 \mathrm{~h}$ of incubation and then suspended in FBS-free DMEM medium. In total, the upper chambers were filled with $200 \mu \mathrm{L}$ suspension containing $5 \times 10^{4}$ cells, while the bottom chambers were added with $500 \mu \mathrm{L}$ of DMEM containing $20 \%$ FBS. After incubation for $24 \mathrm{~h}$, the cells that invaded through the membrane were fixed with $4 \%$ paraformaldehyde and stained by $0.5 \%$ crystal violet. Finally, the non-invaded cells were cleared, and the invaded cells were photographed under an inverted microscope (Olympus IX83; Olympus Corporation, Tokyo, Japan). Five random fields of each chamber were selected for quantification.

\section{Tumor xenograft assay}

miR-129-2-3p mimics or miR-NC-transfected cells were subcutaneously injected into the upper flank of 4-5 weeks old nude mice (Shanghai Laboratory Animal Center; Shanghai, China).The size of tumor xenograft was read every week. All of the nude mice were sacrificed four weeks after seeding. The tumor xenografts were resected and weighed. Tumor volume was analyzed using the following formula: tumor volume $(\mathrm{mm} 3)=\left(\right.$ length $\times$ width $\left.{ }^{2}\right) / 2$. All experimental procedures were approved by the Ethics Committee of Hunan Provincial People's Hospital/The First Affiliated Hospital of Hunan Normal University.

\section{Bioinformatics analysis and luciferase reporter assay}

TargetScan 7.1 (http://www.targetscan.org/) and miRanda (http://www.microrna.org) were employed to search for the putative targets of miR-129-2-3p. The 3'-UTR regions of human Wip1 gene containing the predicted wild-type (wt) or mutant (mut) miR-129-2-3p binding sites were synthesized by Shanghai GenePharma Co., Ltd. The 3'-UTR fragments were then inserted into the pMIR-REPORT vector (Promega, Madison, WI, USA) to generate the reporter plasmids, pMIR-Wip1-3'UTR-wt and pMIR-Wip1-3'-UTR-mut. Luciferase reporter assay was performed as follows: cells were inoculated into 24-well plates, then co-transfected with miR-129-2-3p mimics or miR-NC and pMIR-Wip1-3'-UTR-wt or pMIR-Wip1-3'-UTR-mut, using Lipofectamine 2000. Following $48 \mathrm{~h}$ incubation, transfected cells were collected, and the luciferase activity was determined using a dualluciferase reporter assay system (Promega, Madison, WI, USA). The firefly luciferase activity was normalized to Renilla luciferase activity.

\section{Protein extraction and western blot analysis}

Tissues or cells were solubilized using the active protein extraction kit (KGP1050; Nanjing KeyGen Biotech Co., Ltd., Nanjing, China) supplemented with protease inhibitors (Millipore, Billerica, MA). The concentration of total protein was detected by using an Enhanced BCA Protein Assay Kit (Beyotime Institute of Biotechnology, Shanghai, China). Equivalent of total protein was loaded for SDS-PAGE on $10 \%$ polyacrylamide gels, followed by transferring to PVDF membranes (Millipore, Billerica, MA) and blocking with $5 \%$ skimmed milk for $2 \mathrm{~h}$. The membranes were then incubated overnight at $4^{\circ} \mathrm{C}$ with primary antibodies against Wip1 (cat. no. sc-517220; Santa Cruz Biotechnology, CA, USA) or GAPDH (cat. no. ab125247; Abcam, Cambridge, MA, USA). Subsequently, the membranes were incubated with horseradish peroxidase-conjugated immunoglobulin G goat anti-mouse (cat. no. ab6789; 1:5,000 dilution; Abcam) secondary antibodies at room temperature for $2 \mathrm{~h}$. Immunoreactivity was visualized by using an enhanced chemiluminescence reagents (ECL; Pierce; Thermo Fisher Scientific, Inc.).

\section{Statistical analysis}

All the data were expressed as mean values \pm standard deviation, and subjected to Social Sciences (SPSS) version.16.0 (SPSS, Inc., Chicago, IL, USA) for data analysis. Student's t-test was used to compare the difference between two groups. Comparison among multiple groups was performed using a one-way analysis of variance (ANOVA) test followed by a Bonferroni's post hoc test. The correlation between miR-129-2-3p and clinicopathological characteristics in ICC patients was assessed using chi-square test. Spearman's correlation analysis was adopted to analyze the correlation between miR-129-2-3p and Wip1 mRNA levels in ICC tissues. The differences were defined as statistically significant if $P$ value less than 0.05 . 
A

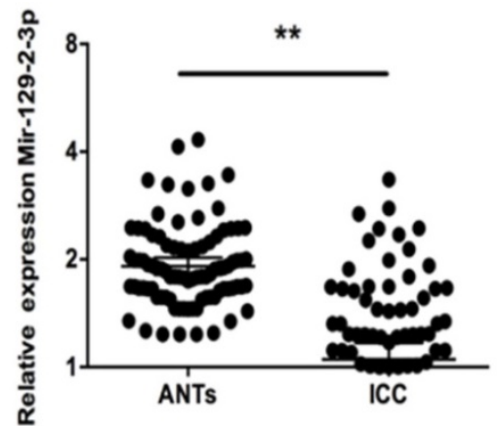

B

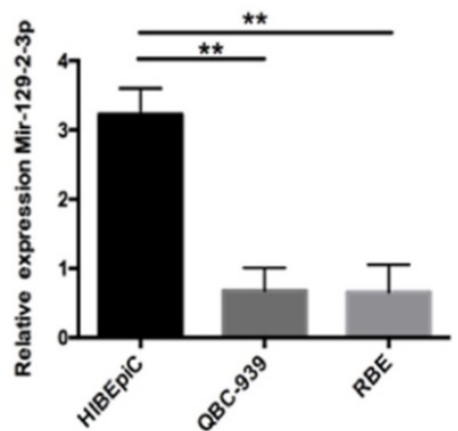

Figure 1. miR-129-2-3p is downregulated in ICC tissues and cell lines. (A) The expression level of miR-129-2-3p was assessed in 90 pairs of ICC tissues and adjacent normal tissues (ANTs) using RT-qPCR. *P<0.05 vs. ANTs. (B) RT-qPCR was carried out to measure miR-129-2-3p expression in the normal human biliary epithelial cell (BEC) and two ICC cell lines (RBE and QBC-939). ${ }^{* * P}<0.01$ vs.BEC

Table 1. Clinicopathological correlation with miR-129-2-3p expression

\begin{tabular}{|c|c|c|c|}
\hline \multirow[t]{2}{*}{ Variable } & \multicolumn{2}{|c|}{ miR-129-2-3p } & \multirow[t]{2}{*}{$\mathrm{P}$} \\
\hline & Low $n=74$ & high, $\mathrm{n}=16$ & \\
\hline Age $(Y)(<50 / \geq 50)$ & $29 / 45$ & $5 / 11$ & 0.553 \\
\hline $\mathrm{M} / \mathrm{F}$ & $42 / 32$ & $10 / 6$ & 0.673 \\
\hline Complicated bile duct stone $+/-$ & $38 / 36$ & $3 / 13$ & 0.65 \\
\hline Tumor size $(\mathrm{cm})(<5 / \geq 5)$ & $35 / 39$ & $13 / 3$ & 0.297 \\
\hline Well/moderate/poorly differentiation & $12 / 23 / 39$ & $10 / 4 / 2$ & 0.037 \\
\hline Pathology $\mathrm{T}(\mathrm{T} 1+2) /(\mathrm{T} 3+4)$ & $19 / 55$ & $10 / 6$ & 0.006 \\
\hline Pathology N $0 / 1$ & $21 / 53$ & $14 / 2$ & 0.042 \\
\hline Pathology M 0/1 & $19 / 55$ & $13 / 3$ & 0.01 \\
\hline
\end{tabular}

\section{Results}

\section{miR-129-2-3p expression is decreased in ICC tissues and cell lines}

First of all, RT-qPCR was used for the detection of miR-129-2-3p expression in ICC tissues and adjacent normal tissues (ANTs) obtained from 90 patients with ICC. miR-129-2-3p expression was notably decreased in ICC tissues when compared with that in ANTs (Figure $1 \mathrm{~A}, \mathrm{P}<0.05$ ). The expression level of miR-129-2-3p was also determined in four ICC cell lines, including QBC-939 and RBE. The normal human biliary epithelial cell (BEC) acted as the control. The data indicated that expression level of miR-129-2-3p was lower in the ICC cell lines than that in BEC (Figure 1B, $\mathrm{P}<0.05$ ). A total of 90 ICC patients were subdivided into either low or high miR-129-2-3p expression groups according to the median value as a cutoff (Table 1). Lowly expressed miR-129-2-3p was frequently associated with differentiation $(\mathrm{P}=0.037)$ and pathology $\mathrm{T}(\mathrm{P}=0.006)$. Furthermore, these data also demonstrated that miR-129-2-3p expression was dramatically associated with pathology N ( $\mathrm{P}=0.042)$ and pathology $M \quad(P=0.01)$. These observations indicated that miR-129-2-3p expression may be associated with the development of ICC.

miR-129-2-3p upregulation suppresses the proliferative and invasive abilities of ICC cells

To investigate the functions of miR-129-2-3p in ICC progression, we transfected the miR-129-2-3p mimics into QBC-939 and RBE cells and then transfection efficiency was evaluated using RT-qPCR (Figure 2A, P<0.05). CCK-8 and transwell cell invasion assays were utilized to determine the differences between QBC-939 and RBE cells after transfection with miR-129-2-3p mimics or miR-NC. The miR-129-2-3p overexpressing-QBC-939 and RBE cells exhibited weaker proliferative (Figure 2B, $\mathrm{P}<0.05$ ) and invasive (Figure $2 \mathrm{C}, \mathrm{P}<0.05$ ) abilities in comparison with that in cells transfected with miR-NC. These data implied that miR-129-2-3p exerts tumor suppressive roles in ICC by decreasing cell proliferation and invasion in vitro.

\section{Wip 1 is a direct target gene of miR-129-2-3p in ICC cells}

To gain insight into the mechanisms through which miR-129-2-3p modulates the proliferation and invasion of ICC cells, bioinformatics analysis was performed to predict the putative targets of miR-129-2-3p and found that the 3'-UTR of Wip1 contains a highly conserved binding site for miR-129-2-3p (Figure 3A). Luciferase reporter assay was carried out to explore whether miR-129-2-3p was able to directly bind to the 3'-UTR of Wip1 in ICC cells. Resumption of miR-129-2-3p expression significantly suppressed the luciferase activity of pMIR-Wip1-3'-UTR-wt in QBC-939 and RBE cells $(\mathrm{P}<0.05)$; however, the inhibitory effect was not observed in cells with pMIR-Wip1-3'-UTR-mut (Figure 3B). We next increased miR-129-2-3p expression in QBC-939 and RBE cells to investigate 
whether the expression levels of Wip1 were altered in response. Introduction of miR-129-2-3p evidently reduced Wip1 mRNA (Figure $3 \mathrm{C}, \mathrm{P}<0.05$ ) and protein (Figure 3D, $\mathrm{P}<0.05$ ) expression levels in QBC-939 and RBE cells. Furthermore, we detected Wip1 expression in ICC tissues and ANTs, revealing that expression level of Wip1 mRNA was higher in ICC tissues than that in ANTs (Figure 3E, P<0.05). Taken together, Wip1 is a direct target gene of miR-129-2-3p in ICC cells.

\section{Silenced Wip 1 expression restricts cell proliferation and invasion in ICC}

To explore the functions of Wip1 in OS, si-Wip1 was utilized to silence endogenous Wip1 expression in QBC-939 and RBE cells. The protein level of Wip1 was evidently silenced in QBC-939 and RBE cells after si-Wip1 transfection, as revealed by western blot analysis (Figure $4 \mathrm{~A}, \mathrm{P}<0.05$ ). The results of CCK-8 and transwell cell invasion assays indicated that the proliferation (Figure 4B, P<0.05) and invasion (Figure $4 C, P<0.05)$ of QBC-939 and RBE cells was suppressed after Wip1 depletion. Consequently, silencing Wip1 expression exhibited similar effects as miR-129-2-3p overexpression in ICC cells, further suggesting Wip1 as a function target of miR-129-2-3p in ICC cells.

\section{Wip 1 restoration markedly rescues the miR-129-2-3p-mediated inhibition of cell proliferation and invasion in ICC}

To further clarify whether decreasing Wip1 expression by miR-129-2-3p upregulation was responsible for the restriction of ICC cell proliferation and invasion, we restored Wip1 expression in miR129-2-3p overexpressing-QBC-939 and RBE cells via cotransfection with Wip1 overexpression plasmid pCMV-Wip1 (Figure 5A, P<0.05). In addition, functional experiments revealed that the inhibitory effects of miR-129-2-3p upregulation in QBC-939 and RBE cell proliferation (Figure $5 \mathrm{~B}, \mathrm{P}<0.05$ ) and invasion (Figure $5 \mathrm{C}, \mathrm{P}<0.05$ ) were partially rescued by Wip1 restoration. In summary, Wip1 is a downstream mediator in the tumor suppressive roles of miR$129-2-3 p$ in ICC cells.
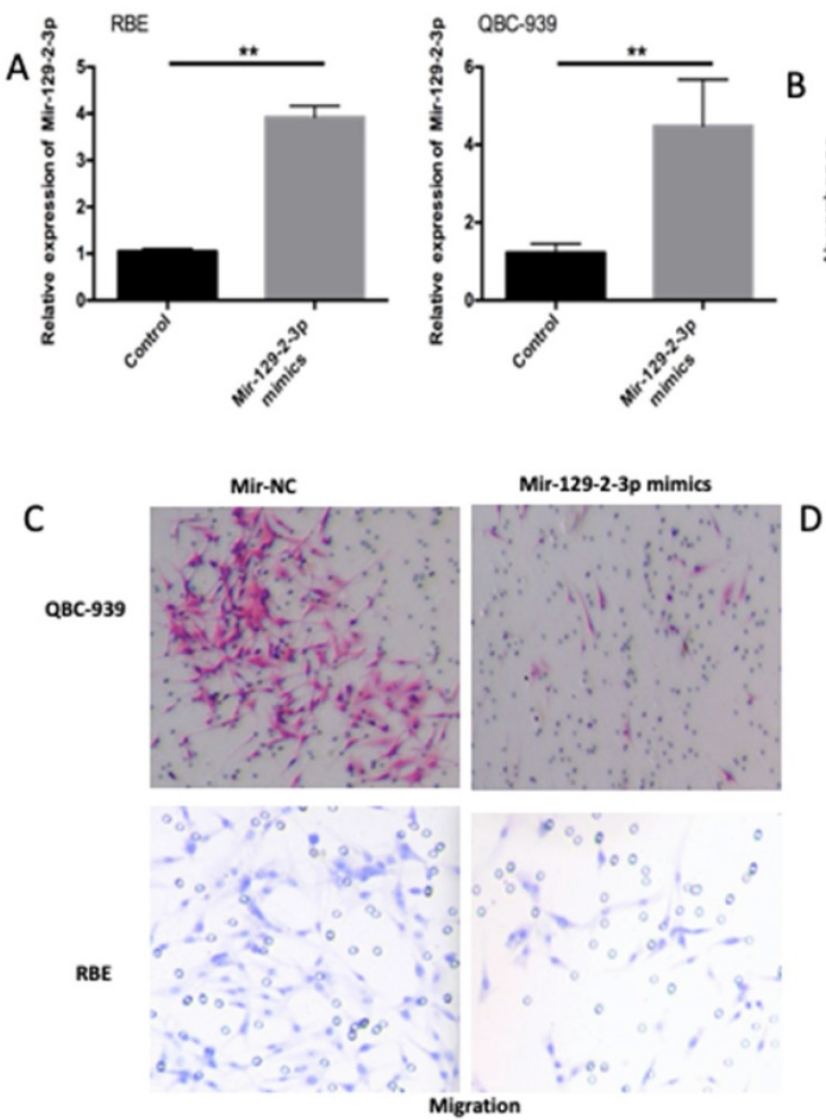
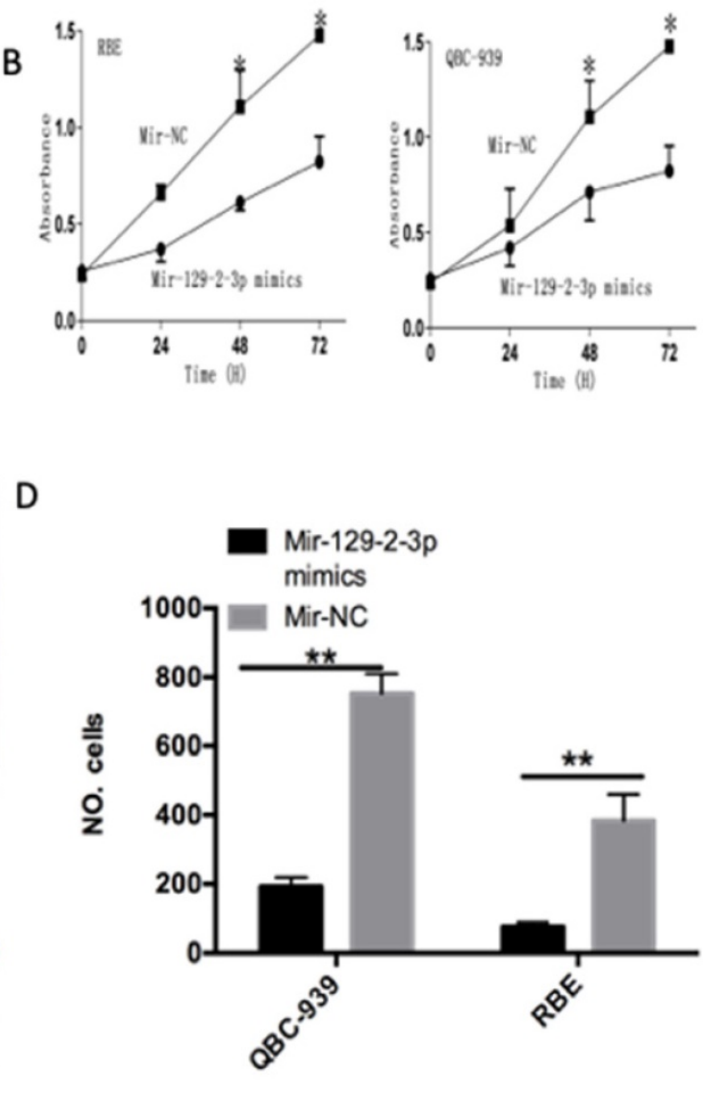

Figure 2. Resumption of miR-129-2-3p expression restrains the proliferation and invasion of RBE and QBC-939 cells. (A) The expression level of miR-129-2-3p was measured in RBE and QBC-939 cells after miR-129-2-3p mimics or miR-NC transfection. $* * P<0.01$ vs. miR-NC. (B) CCK-8 assay was performed to determine the proliferation in miR-129-2-3p mimics or miR-NC-transfected RBE and QBC-939cells. *P<0.05 vs. miR-NC. (C, D) The effect of miR-129-2-3p upregulation on RBE and $\mathrm{QBC}-939$ cell invasion was explored using transwell cell invasion assay. ${ }^{* *} \mathrm{P}<0.01$ vs. miR-NC. 

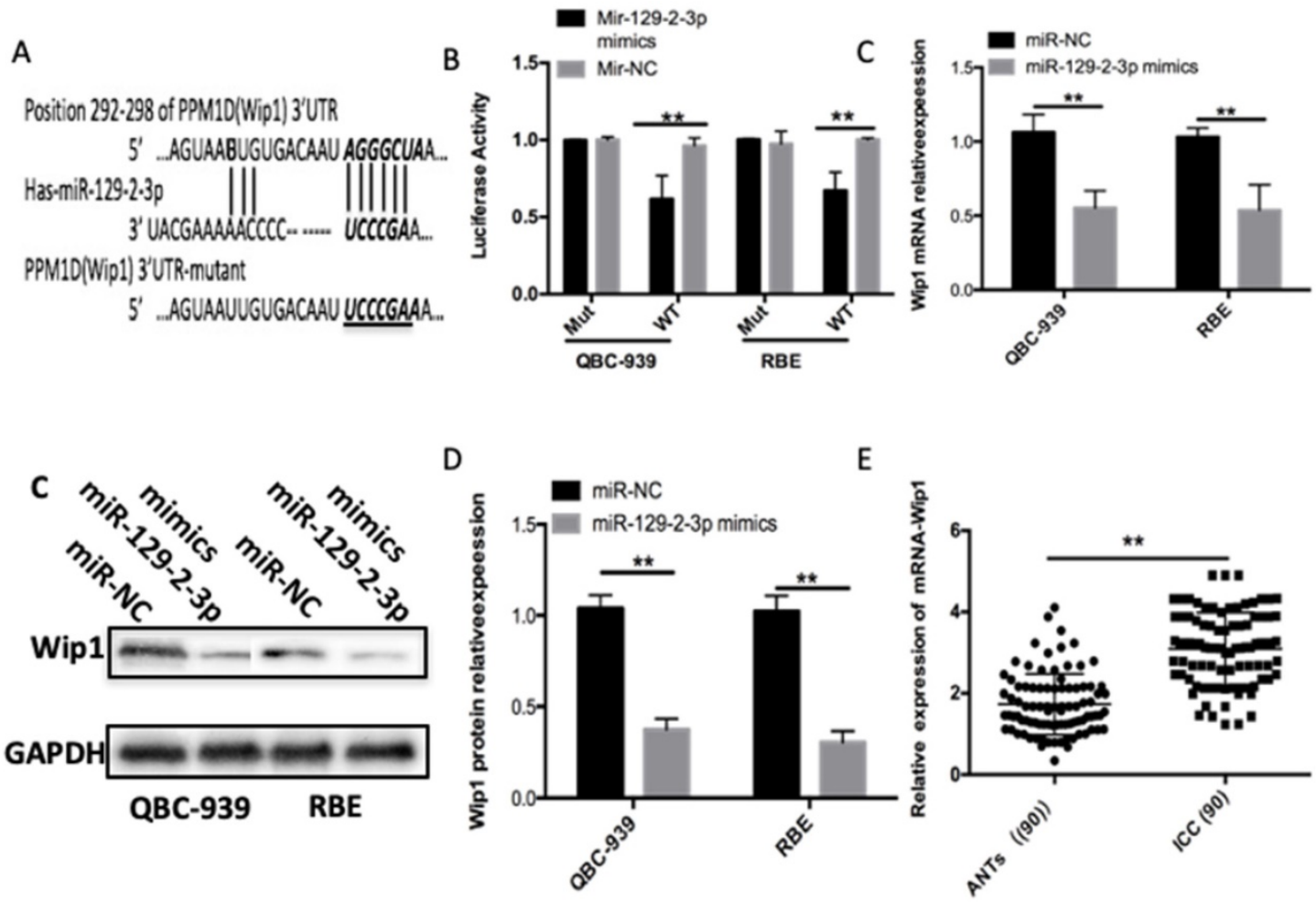

Figure 3. Wipl is a direct target gene of miR-1 29-2-3p in ICC cells. (A) The 3'-UTR of the Wipl contains a potential miR-129-2-3p binding site. The mutant 3'-UTR region of the Wipl is also shown. (B) PMIR-Wipl-3'-UTR-wt or pMIR-Wipl-3'-UTR-mut along with miR-129-2-3p mimics or miR-NC was introduced into RBE and QBC-939cells. After $48 \mathrm{~h}$ culture, luciferase reporter assay was utilized for the measurement of the luciferase activity. $* * P<0.01$ vs. miR-NC. (C, D) RBE and QBC-939cells were transfected with miR-129-2-3p mimics or miR-NC. The mRNA and protein levels of Wipl were measured by RT-qPCR and western blot analysis, respectively. *P<0.05 vs. miR-NC. (E) The mRNA expression of Wipl was detected in 90 pairs of ICC tissues and ANTs using RT-qPCR. **P<0.01 vs. ANTs.

A

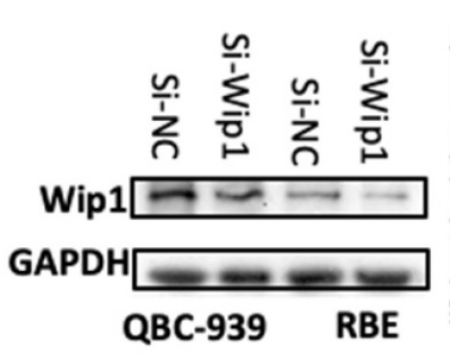

B

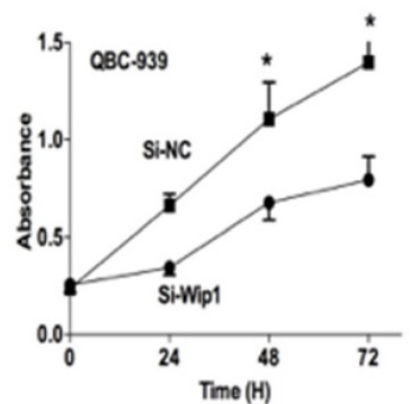

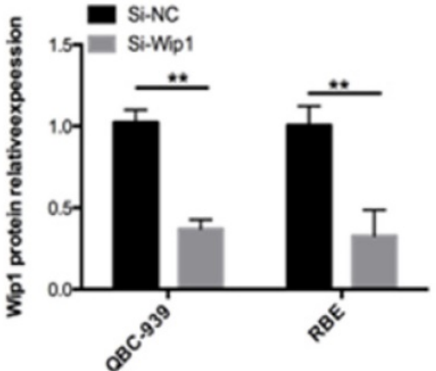

C

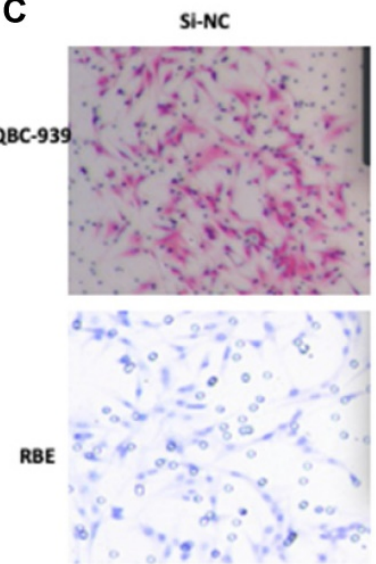

migration

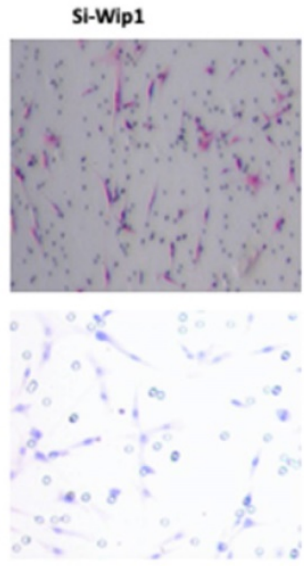

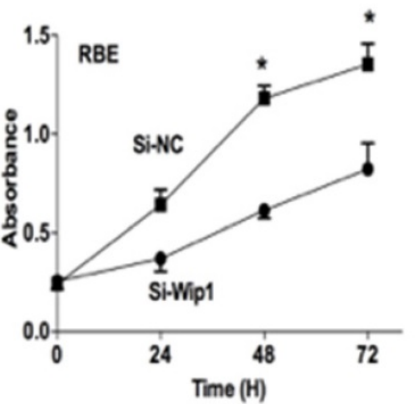

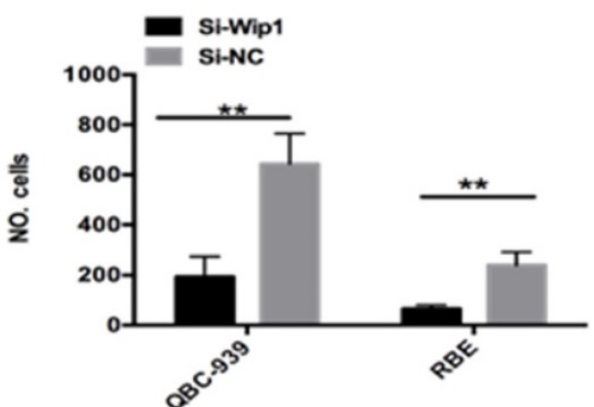

Figure 4. Knockdown of Wipl suppresses the proliferation and invasion of RBE and QBC-939cells. RBE and QBC-939cells were transfected with si-Wipl or si-NC. (A) $72 \mathrm{~h}$ after transfection, the protein level of Wipl was determined by western blot analysis. $* *$ P $<0.01 \mathrm{vs}$. si-NC. (B, C) The proliferation and invasion was assessed by CCK-8 and transwell cell invasion assays, respectively. Wipl-silencing evidently inhibited the abilities of proliferative and invasive in RBE and QBC-939cells. **P $<0.01$ vs. si-NC. 
A

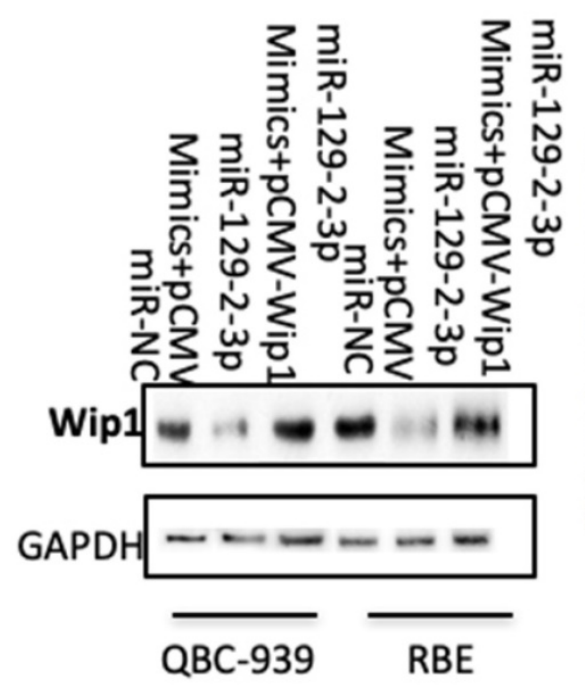

B

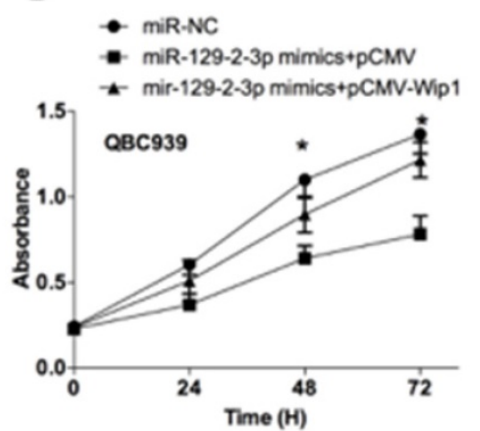

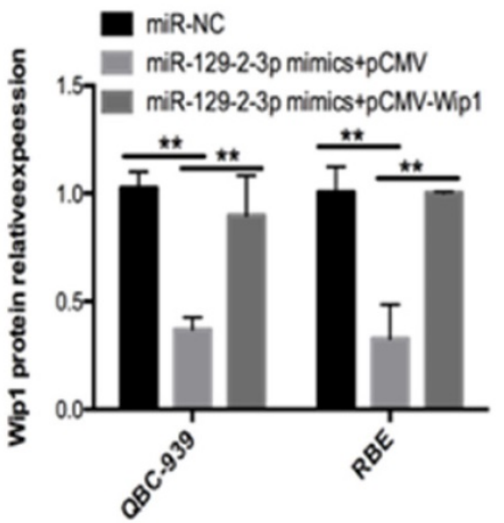

C
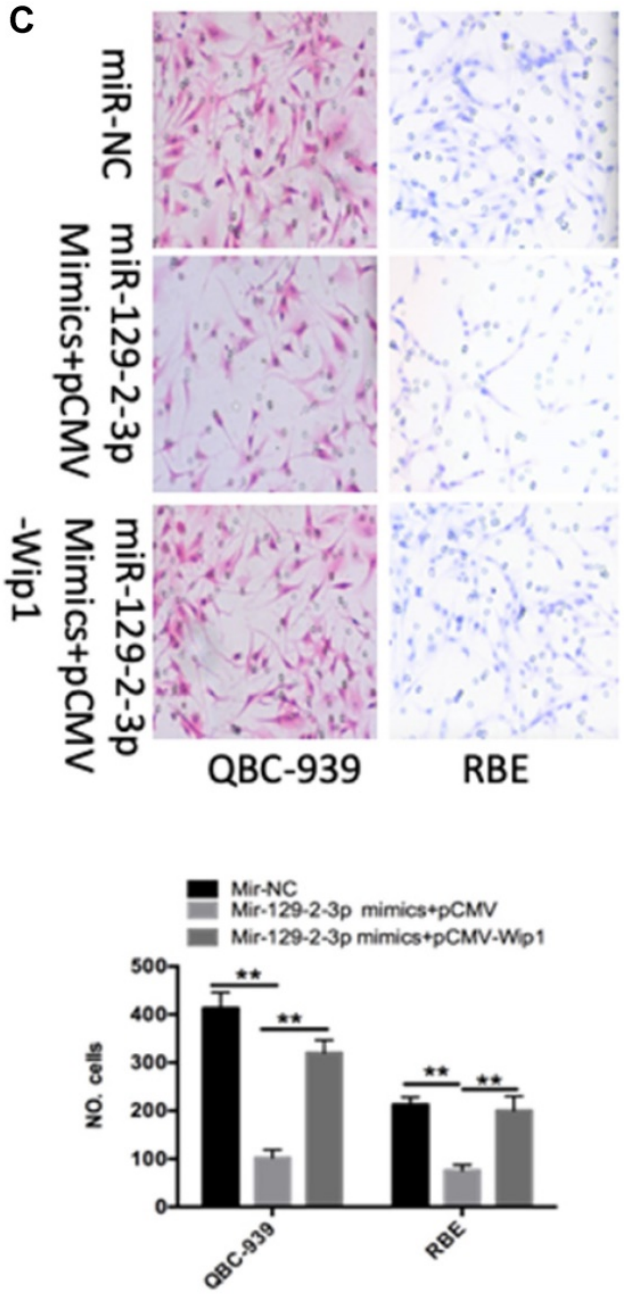

Figure 5. Wipl is required for miR-129-2-3p-directed inhibition of RBE and QBC-939cell proliferation and invasion. miR-129-2-3p-overexpressing RBE and QBC-939cells were transfected with pCMV-WIPI or pCMV. (A) Transfected cells were collected after $72 \mathrm{~h}$ of incubation and subjected to western blot analysis for Wipl protein expression. $* * P<0.01$ vs. miR-NC. $* * P<0.01$ vs. miR-129-2-3p mimics $+p C M V$. $(B, C)$ The proliferation and invasion of RBE and $Q B C-939 c e l l s$ treated as described was investigated by CCK-8 and transwell cell invasion assays, respectively. $* * P<0.01$ vs. miR-NC. $* * P<0.01$ vs. miR-129-2-3p mimics $+p C M V$.

\section{miR-129-2-3p impairs the ICC tumor growth in vivo}

Tumor xenograft assay was performed to assess the influence of miR-129-2-3p on cell tumorigenicity in vivo. ICC cells transfected with miR-129-2-3p mimics were inoculated into nude mice, and miR-NC-transfected cells were used as a control. The volume and weight of tumor xenograft derived from miR-129-2-3p mimics-transfected ICC cells was notably smaller (Figures $6 \mathrm{~A}$ and $\mathrm{B}, \mathrm{P}<0.05$ ) and lighter (Figure $6 \mathrm{C}, \mathrm{P}<0.05$ ) than that in the miR-NC groups. Next, RT-qPCR was utilized for the detection of miR-129-2-3p expression in the tumor xenograft. Higher miR-129-2-3p expression was observed in the tumor xenograft of miR-129-2-3p mimics group compared with that in the miR-NC group (Figure 6D, $\mathrm{P}<0.05)$. Furthermore, the protein level of Wip1 in the tumor xenograft was determined via western blot analysis. Results revealed that the expression of Wip1 protein was evidently downregulated in the
miR-129-2-3p mimics-treated nude mice group (Figure 6E, $\mathrm{P}<0.05$ ). These results implied that miR-129-2-3p impaired the tumor growth of ICC cells in vivo.

\section{Discussion}

Intrahepatic cholangiocarcinoma (ICC) is the second most common primary hepatic malignancy with poor prognosis $[17,20]$. Despite improvements in its diagnosis and therapy, the prognosis for ICC patients remains poor. An improved understanding of ICC tumorigenicity and consequential identification of novel therapeutic targets would improve the prognosis of ICC patients [17]. MicroRNAs (miRNAs) are a class of highly conserved, small endogenous non-coding RNAs that regulate gene expression at the posttranscriptional level and participate in important cellular processes, including development, apoptosis, proliferation, differentiation, metabolism $[9,21]$. Several studies 


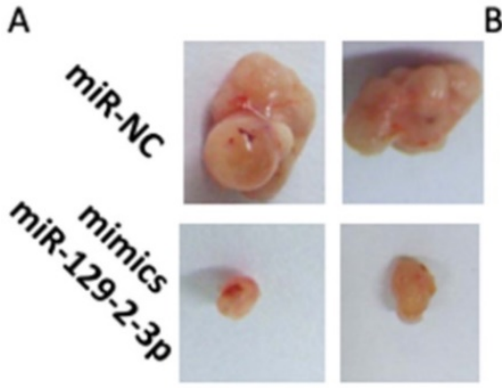

E

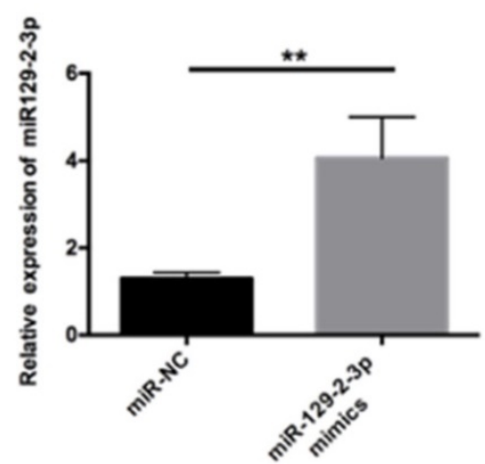

$\mathrm{F}$
B
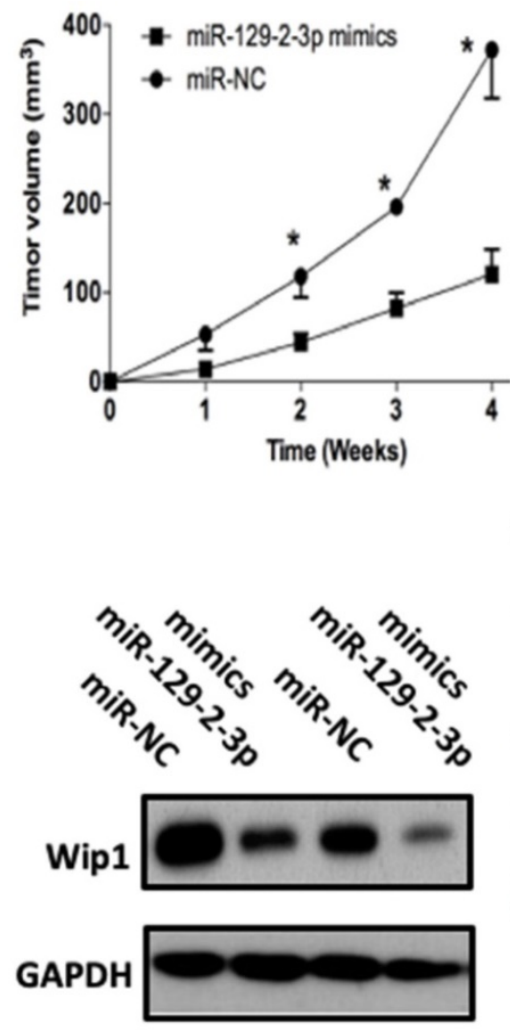

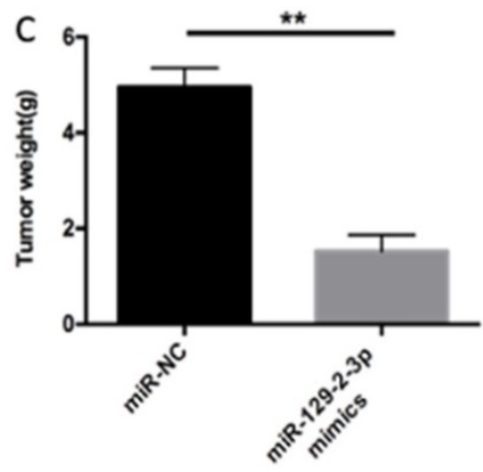

G

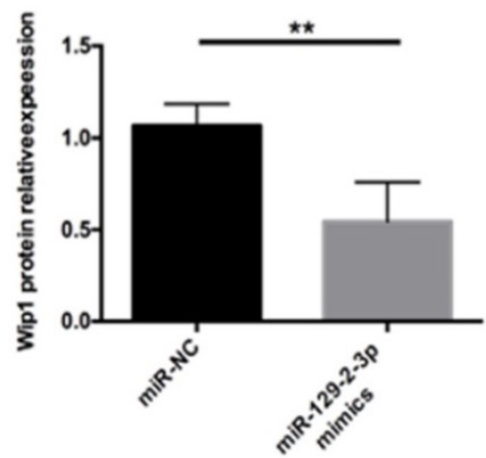

Figure 6. miR-129-2-3p upregulation impairs ICC tumor growth in vivo. (A) Representative images of tumor xenografts derived from $Q B C-939$ cells transfected with miR-129-2-3p mimics or miR-NC. (B) The volume of tumor xenografts in the miR-129-2-3p mimics group was smaller than that in the miR-NC group. *P<0.05 compared with miR-NC. (C) The tumor xenografts in the miR-129-2-3p mimics and miR-NC group were excited and weighed after four weeks of implantation. **P<0.01 vs. miR-NC. (D) RT-qPCR was performed to analyze miR-129-2-3p expression in the tumor xenografts. **P<0.01 vs. miR-NC. (E) The expression level of Wipl protein in tumor xenografts was analyzed by western blot analysis. ${ }^{*} * \mathrm{P}<0.01$ vs. miR-NC.

have demonstrated downregulated expression of miRNAs in ICC tissues and cell lines, in which these miRNAs exert important roles in ICC apoptosis, cell proliferation, invasion and migration $[9,21]$. In this paper, we elucidate the potential role of miR-129-2-3p in the pathogenesis of ICC and investigate the possibilities of using miR-129-2-3p as diagnostic and prognostic marker, as well as therapeutic target in ICC.

The miR-129 family includes two precursors miR-129-1 and miR-129-2 which are processed to three mature miRNAs, miR-129-5p, miR-129-1-3p and miR-129-2 [21]. Few studies have investigated the roles of the miR-129 family members in cell proliferation and metastasis in ICC. Dual functions for miR-129, as a tumor suppressor and oncogene, have been confirmed in various types of carcinomas. miR-129-5p has been reported to be downregulated in neuroendocrine tumors, prostate cancer, lung cancer and gastric cancer and functions as a tumorsuppressor role in these carcinomas [22-24]. Epigenetic regulation of miR-129-2 was demonstrated in glioma and lung cancer [25,26]. In contrast, however, miR-129 is also upregulated in several solid tumors and non-cancerous tissues from cancer patients with lymph node metastases [27-29].
Our previous findings indicate that Wip1 is involved in the tumorigenicity and invasion of human ICC at least in part through the MMP-2 signaling pathway [17]. Our further data show that both iNOS and Wip1 promote ICC cell migration and invasion by up-regulating MMP expression [18]. To evaluate the underlying mechanism, we find miR-129-2-3p directly targets wip1 to suppress the proliferation and invasion of intrahepatic cholangiocarcinoma.

Wip1, located on chromosome 17q22-q23, acts as an oncogene and inhibits p53 activity when expressed at high levels along with oncogenes [30]. These features are mainly connected with Wip1 ability to regulate DNA damage response (DDR) signaling and MAPK kinases pathway p53 network, including p38, p53, ATM, Chk2, and $\gamma$-H2AX [10,31,32]. Accumulating studies identified that high Wip1 expression disrupted the homeostasis maintained by the p38 MAPK-p53-Wip1 pathway, which caused downstream Wnt-p53 inactivation through p38 MAPK dephosphorylation, and promoted the development of malignant in humans by reducing p16 protein levels [33,34]. Our previous data have indicated that Wip1 is oncogenic and is involved in invasive growth in renal cancer cells and ICC cells $[12,17]$. Our data also show Wip1 involved in the 
tumorigenicity and invasion of human ICC in part through the MMP-2 signaling pathway [17]. In this study, we found that miR-129-2-3p was the upstream regulator of Wip1.

In summary, our results revealed that miR-129-2-3p expression was decreased in ICC tissues and cells. A low miR-129-2-3p expression was significantly correlated with malignant clinical features of patients with ICC. Additionally, we have shown, for the first time, that miR-129-2-3p exerted tumor suppressive roles in ICC progression through its target gene Wip1. Hence, this study provided functional evidence that fully supports the hypothesis that miR-129-2-3p might be a promising target for the management of patients with ICC.

\section{Acknowledgments}

\section{Funding}

This work was financially supported by following funds: National science founding of china (81902017)/Hunan Provincial Natural Science Foundation of China (Grant No. 2019JJ50320/ 2019JJ20011)/Central Guidance of Local Science and Technology Development Fund (Grant No. 2018CT5008)/Project of Scientific Research of Traditional Chinese Medicine in Hunan (Grant No. 201809)/Hunan province technical innovation guidance program-clinical medical technology innovation guidance project (2018SK50708)/Clinical Research Center For Anesthesiology of ERAS in Hunan Province (No. 2018SK7001)/Fund of the Hunan provincial health and family planning commission (C2016005).

\section{Competing Interests}

The authors have declared that no competing interest exists.

\section{References}

1. Mavros MN, Economopoulos KP, Alexiou VG, et al. Treatment and Prognosis for Patients With Intrahepatic Cholangiocarcinoma: Systematic Review and Meta-analysis. JAMA Surg. 2014; 149: 565-574.

2. Wang Y, Li J, Xia Y, et al. Prognostic nomogram for intrahepatic cholangiocarcinoma after partial hepatectomy. J Clin Oncol. 2013; 31: 1188-1195.

3. Shaib YH, Davila JA, McGlynn K, et al. Rising incidence of intrahepatic cholangiocarcinoma in the United States: a true increase? J Hepatol. 2004; 40: 472-477.

4. Doussot A, Lim C, Gomez Gavara C, et al. Multicentre study of the impact of morbidity on long-term survival following hepatectomy for intrahepatic cholangiocarcinoma. Br J Surg. 2016; 103: 1887-1894..

5. Ghiassi-Nejad Z, Moshier E, Schwartz M, et al. Definitive Chemoradiation Therapy in the Treatment of Unresectable Intrahepatic Cholangiocarcinoma: A National Cancer Data Base Study. Int J Radiat Oncol Biol Phys. 2016; 96: E211-E212.

6. Li Z, Shen J, Chan MT, et al. The role of microRNAs in intrahepatic cholangiocarcinoma. J Cell Mol Med. 2017; 21: 177-184.

7. Yu X, Luo L, Wu Y, et al. Gastric juice miR-129 as a potential biomarker for screening gastric cancer. Med Oncol. 2013; 30: 365.

8. Yu X, Song H, Xia T, et al. Growth inhibitory effects of three miR-129 family members on gastric cancer. Gene. 2013; 532: 87-93.

9. Wang D, Luo L, Guo J. miR-129-1-3p inhibits cell migration by targeting BDKRB2 in gastric cancer. Med Oncol. 2014; 31: 98.
10. Brazina J, Svadlenka J, Macurek L, et al. DNA damage-induced regulatory interplay between DAXX, p53, ATM kinase and Wip1 phosphatase. Cell Cycle. 2015; 14: 375-387.

11. Chong KH, Samarasinghe S, Kulasiri D. Mathematical modelling of p53 basal dynamics and DNA damage response. Math Biosci. 2015; 259: 27-42.

12. Liu S, Qi L, Han W, et al. Overexpression of wip1 is associated with biologic behavior in human clear cell renal cell carcinoma. PLoS One. 2014; 9: e110218.

13. Emelyanov A, Bulavin DV. Wip1 phosphatase in breast cancer. Oncogene. 2015; 34: 4429-4438.

14. Fu Z, Sun G, Gu T. Proto-oncogene Wip1, a member of a new family of proliferative genes in NSCLC and its clinical significance. Tumour Biol. 2014; 35: 2975-2981

15. Yin S, Wang P, Yang L, et al. Wip1 suppresses ovarian cancer metastasis through the ATM/AKT/Snail mediated signaling. Oncotarget. 2016; 7: 29359-29370.

16. Richter M, Dayaram T, Gilmartin AG, et al. WIP1 phosphatase as a potential therapeutic target in neuroblastoma. PLoS One. 2015; 10: e0115635.

17. Liu S, Jiang B, Li H, et al. Wip1 is associated with tumorigenity and metastasis through MMP-2 in human intrahepatic cholangiocarcinoma. Oncotarget. 2017; 8: $56672-56683$.

18. Liu SL, Jiang JQ, Huang LS, et al. iNOS is associated with tumorigenicity as an independent prognosticator in human intrahepatic cholangiocarcinoma. Cancer Management and Research. 2019; 11: 8005-8022.

19. Livak KJ, Schmittgen TD. Analysis of relative gene expression data using real-time quantitative PCR and the 2(-Delta Delta C(T)) Method. Methods. 2001; 25: 402-408.

20. Goeppert B, Toth R, Singer S, et al. Integrative analysis defines distinct prognostic subgroups of intrahepatic cholangiocarcinoma. Hepatology. 2019; 69: 2091-2106.

21. Tang X, Tang J, Liu X, et al. Downregulation of miR-129-2 by promoter hypermethylation regulates breast cancer cell proliferation and apoptosis. Oncol Rep. 2016; 35: 2963-2969.

22. Gao G, Xiu D, Yang B, et al. miR-129-5p inhibits prostate cancer proliferation via targeting ETV1. Onco Targets Ther. 2019; 12: 3531-3544.

23. Wang S, Chen $\mathrm{Y}, \mathrm{Yu} \mathrm{X}$, et al. miR-129-5p attenuates cell proliferation and epithelial mesenchymal transition via HMGB1 in gastric cancer. Pathol Res Pract. 2019; 215: 676-682.

24. Li G, Xie J, Wang J. Tumor suppressor function of miR-129-5p in lung cancer. Oncol Lett. 2019; 17: 5777-5783.

25. Trujillo-Gonzalez I, Wang Y, Friday WB, et al. MicroRNA-129-5p is regulated by choline availability and controls EGF receptor synthesis and neurogenesis in the cerebral cortex. FASEB J. 2019; 33: 3601-3612

26. Ames HM, Yuan M, Vizcaino MA, et al. MicroRNA profiling of low-grade glial and glioneuronal tumors shows an independent role for cluster $14 \mathrm{q} 32.31$ member miR-487b. Mod Pathol. 2017; 30: 204-216.

27. Chen Y, Huang W, Sun W, et al. LncRNA MALAT1 Promotes Cancer Metastasis in Osteosarcoma via Activation of the PI3K-Akt Signaling Pathway. Cell Physiol Biochem. 2018; 51: 1313-1326.

28. Fu $Q$, Jiang $H$, Wang $Z$, et al. Injury factors alter miRNAs profiles of exosomes derived from islets and circulation. Aging (Albany NY). 2018; 10: 3986-3999.

29. Umehara $T$, Mori $R$, Mace $K A$, et al. Identification of Specific miRNAs in Neutrophils of Type 2 Diabetic Mice: Overexpression of miRNA-129-2-3p Accelerates Diabetic Wound Healing. Diabetes. 2019; 68: 617-630.

30. Fiscella M, Zhang H, Fan S, et al. Wip1, a novel human protein phosphatase that is induced in response to ionizing radiation in a p53-dependent manner. Proc Natl Acad Sci U S A. 1997; 94: 6048-6053.

31. Lin J, Zhang Q, Lu Y, et al. Downregulation of HIPK2 increases resistance of bladder cancer cell to cisplatin by regulating Wip1. PLoS One. 2014; 9: e98418.

32. Elias J, Dimitrio L, Clairambault J, et al. The dynamics of p53 in single cells: physiologically based ODE and reaction-diffusion PDE models. Phys Biol. 2014; 11: 045001.

33. Goloudina AR, Kochetkova EY, Pospelova TV, et al. Wip1 phosphatase: between p53 and MAPK kinases pathways. Oncotarget. 2016; 7: 31563-31571.

34. Pechackova S, Burdova K, Benada J, et al. Inhibition of WIP1 phosphatase sensitizes breast cancer cells to genotoxic stress and to MDM2 antagonist nutlin-3. Oncotarget. 2016; 7: 14458-14475. 